\title{
Physics major engagement and persistence: a phenomenography interview study
}

\author{
Eric A. Williams, ${ }^{1}$ Justyna P. Zwolak, ${ }^{2,3, *}$ and Eric Brewe ${ }^{4,5}$ \\ ${ }^{1}$ Department of Physics, Florida International University, Miami, Florida 33199 \\ ${ }^{2}$ Department of Teaching and Learning, Florida International University, Miami, Florida 33199 \\ ${ }^{3}$ STEM Transformation Institute, Florida International University, Miami, Florida 33199 \\ ${ }^{4}$ Department of Physics, Drexel University, Philadelphia, PA 19104 \\ ${ }^{5}$ School of Education, Drexel University, Philadelphia, PA 19104
}

\begin{abstract}
Over a ten-year period, physics graduation rates at Florida International University (FIU) increased by about $480 \%$ [1]. To shed light on this phenomenon we conducted an interview study of upper division physics students to learn about their experiences, successes, and challenges. We interviewed 10 students using a semi-structured interview protocol based on the student engagement and persistence work of Tinto and Nora [2, 3]. We then followed Marton's approach to phenomenography to code and analyze the recorded interviews. Phenomenography is defined as "a research method for mapping the qualitatively different ways in which people experience, conceptualize, perceive, and understand various aspects of, and phenomena in, the world around them" [4]. This pilot analysis describes the engagement and persistence of two physics majors at FIU. Findings suggest that working as a Learning Assistant, integration with a consistent peer group, and social support from fellow students promote physics majors' persistence.
\end{abstract}

\section{INTRODUCTION}

Over a course of a little over 10 years, the FIU physics department experienced rapid growth in the number of declared and intended physics majors: while FIU's undergraduate population as a whole increased by $44 \%$, the number of physics majors grew by approximately $480 \%$ (see Fig. 1 ). Concurrent to this rapid growth, the physics department instituted a variety of interventions: the implementation of a Modeling Instruction course in a newly-renovated classroom, the opening of a dedicated conference room and lounge to form a Physics Learning Center (PLC), and the creation of a Learning Assistant (LA) program. This study is a part of a larger analysis that aims to determine how these interventions affected physics students and identify the important features to which students' successes and challenges may be attributed. In this context, we define success as graduating with a physics degree. This preliminary work investigates the case of two students: one of whom successfully graduated with a physics degree, and one who changed to a non-physics major.

Our theoretical framework was developed from a body of literature that links student engagement to persistence $[2,3,5,6]$. Student engagement is a meta-construct that describes a student's interaction with the academic system on three dimensions: behavioral, emotional, and cognitive [7]. This construct appears in the literature under several names, including involvement [8] and integration [2], which have often been used interchangeably [9]. In this paper, we use these terms to describe the degree to which a student immerses themselves into the social and academic fabric of the university, with special emphasis on the physics community. For this study, we define persistence as the successful completion of a college degree. It should be noted that FIU is an

\footnotetext{
* JPZ currently at QuICS, UMD, College Park, MD 20742, USA and NIST, Gaithersburg, MD 20899, USA
}

\section{FIU Undergraduate Physics Majors}



FIG. 1. Number of Declared and Intended Physics Majors at FIU, 1992-2012 [1].

urban Hispanic-Serving Institution (HSI) where 90\% of students are commuters and more than half are first-generation college students $[10,11]$; our theoretical framework is sensitive to the unique challenges that these students face.

\section{METHODS}

\section{A. Phenomenography}

In our study, we followed Marton's approach to phenomenography, which is characterized by the following features: (1) data are used to learn about a phenomenon or issue; the case is secondary (2) the use of pattern-matching and cross-case synthesis to find emergent themes (3) context is especially important, and the boundary between phenomenon and context may be unclear (4) the analysis is an iterative process, requiring previously coded data to be resorted if subse- 
quent data suggests a new/different scheme (5) the framework develops as the study progresses, based on "the most essential and distinctive characteristics" in the data [4].

It is important to note that in phenomenography the categories are meant to be wholly the end result of the study. Based on the theoretical framework, we had some preconceived categories in mind, especially those suggested in Ref. [3]. However, we stress that we did not attempt to force the statements to fit into the theoretical category structure: we remained open to the creation of new and/or different categories for statements that defy prior findings.

On the other hand, the theoretical framework can serve as a validation technique. Categories identified in the analysis can be compared and contrasted with themes suggested in the theoretical framework, verifying and expanding upon the previously developed categories [2, 3, 12, 13].

\section{B. Interview protocol}

An interview protocol was developed from the theoretical framework, in particular Tinto's work on student integration $[2,5]$ and Nora's model of student engagement and persistence [3]. It was also informed by Crisp et al.'s study of STEM persistence at an Hispanic-Serving Institution [6]. Results from Refs. [13] and [14] were especially important, as they explored specific mechanisms of student engagement particular to FIU. This framework led to questions about students' experiences that were aimed at identifying the defining features of their engagement and determining the key factors that supported or inhibited their persistence. For instance, to gain insight into students' attitudes toward being a physics major, we asked them questions such as "What attracted you to becoming a physics major?", "Have you encountered challenges in majoring in physics? How have you overcome these challenges?", "Have you had doubts about continuing to major in physics? Why/what are they?", and "Describe your career goals." To understand students' involvement in various groups within and outside of the university, we asked questions such as "Do you have people in your major that you socialize with inside/outside of school?", "Are you involved in any on-campus groups? Which ones? Why?" and "What sorts of things are you involved with outside of school (work/volunteering/hobbies/church)?" Follow-up and clarifying questions were used as the interviewer felt necessary.

\section{Data collection}

We used our protocol to interview two senior-level students: one upper division physics major very close to graduating, and one fourth-year student who began as a physics major (BS), changed to biology, came back to the physics program (in education), and ultimately changed again to environmental sustainability. Both of these students are women. Senior-level students are of interest because they can provide holistic, retrospective insight into their defining lived experiences, and how these experiences impacted their persistence. These two students were chosen for the pilot study because of their marked contrast.

The interviews were conducted in a semi-structured format to allow for participants' individual interpretation of the question they were answering and emergence of the points they find salient [4]. Importantly, this format also offered opportunities for the researchers to ask relevant follow-up questions.

Interviews were video and audio recorded for later analysis. The data were stored on a secure hard drive in a locked room pursuant to IRB requirements. Interview participants were presented with consent forms; they were informed that their participation is voluntary as well as about how the data would be used. Participants were not offered any compensation for their time.

\section{Coding the data}

The interview recordings were paraphrase-transcribed by the authors; statements deemed particularly important were transcribed verbatim. Each statement representing a distinct category was written on the front side of an index card. The reverse side was marked with identifying information including which participant said it, the time-stamp, and a unique identification code. This was done to ease grouping and comparison between various statements, while making it possible to track each statement based on respondent and position within the transcript.

The statement cards were laid out on a large table and sorted into groups based on emergent themes. Participants' statements were then analyzed with a phenomenographic approach: statement cards were rearranged iteratively and cyclically, requiring reorganization of the early groups if a later card suggested new/different categories were required. We paid special attention to both the context in which the statement was uttered, and the context of the group into which it was placed.

Participant statements were grouped according to recurrent themes and emergent patterns, which were used to identify a factor that either contributed to or detracted from a student's ability to engage and persist. These factors were then organized into modules such as Individual Characteristics, Environment/Barriers, Interactions with Physics Community, and Other. In what follows, we focus on factors from the Interactions with Physics Community module, while also referring to factors from the Environment/Barriers module that are related.

\section{ANALYSIS AND RESULTS}

We start with a big-picture perspective of the lived experiences of the two students. In particular, we compare their onand off-campus circumstances as well as their self-reported 
TABLE I. Selected factors about student engagement and persistence, and the statements that led to their creation. Certain aspects of these factors help persistence; others hinder it. Place of Residence and Work Responsibility are from the Environment/Barriers module. Consistent Peer Group and Social Support are from the Interactions with Physics Community module. R stands for researcher.

\begin{tabular}{lll}
\hline \hline Factor & Help & Hinder \\
\hline Place of Residence & $\begin{array}{l}\text { Student L lived on campus for 1 year as a Resident } \\
\text { Assistant. }\end{array}$ & $\begin{array}{l}\text { Student L commuted for 6 years. Student S lived } \\
\text { off-campus (30-35 minutes away). }\end{array}$ \\
\hline Work Responsibility & $\begin{array}{l}\text { Student S worked as a physics LA for 1 semester. } \\
\text { Student L worked as a physics LA for 6 semesters. }\end{array}$ & $\begin{array}{l}\text { Student S worked 25 hours per week (off-campus, } \\
\text { 30 minutes away.) }\end{array}$ \\
\hline Consistent Peer Group & $\begin{array}{l}\text { L:"It helped having people there who were seeing } \\
\text { you struggle, and you're asking them questions day } \\
\text { in and day out... they were willing to sit down and } \\
\text { are really good at explaining things." }\end{array}$ & $\begin{array}{l}\text { Student S: "I usually just pick up whoever is in the } \\
\text { class... it's hard to stick with the same people, es- } \\
\text { pecially since you're changing your major around a } \\
\text { billion times [four times]" (lack of consistent peer } \\
\text { group). }\end{array}$ \\
& $\begin{array}{l}\text { L: "It was really cool to have 3 years where I could } \\
\text { hang out with people going through the same things } \\
\text { as me." }\end{array}$ & \\
& $\begin{array}{l}\text { L: "Great to have people like you going through the } \\
\text { same classes, having the same struggles." }\end{array}$ & \\
\hline $\begin{array}{l}\text { R: Did you ever have doubts about continuing in } \\
\text { physics? And how did you over come them? L: } \\
\text { "friends, people helping out working on the home- } \\
\text { work, positive feedback from fellow students" }\end{array}$ & $\begin{array}{l}\text { S: "no really close friends here [at FIU]...I have a } \\
\text { lot of friends from work up in [hometown, 30 min- } \\
\text { utes away]... I'm a little scattered everywhere" }\end{array}$ \\
\hline \hline
\end{tabular}

attitudes toward being a physics major. We pay special attention to their interactions with the physics community, both academic and social. We then move on to a cross-case analysis of the emergent themes. Finally, we compare emergent themes to relevant concepts in the theoretical framework.

\section{A. A tale of two students}

The two students considered in this study had markedly different experiences. Student S arrived at FIU with a physics major pre-declared, but changed majors to biology then to physics education then to environmental sustainability. Concurrent to this, she worked 25 hours per week at an offcampus job and failed to establish long-term social ties on campus; instead, she picked up study partners solely on a class-by-class basis. Notably, she taught as an LA for one semester (thus engaging with a consistent group of physics professors, TAs, and other LAs several times per week) and identified this as a key point of reentry to the physics (education) program, but ultimately left this to pursue a different major. Student S's experience can best be summed up in her own words: "[My job], my dogs, bed, school. That's life." In other words, it sounds as if she did not create the necessary time or space in her life to meaningfully integrate within the social fabric of the physics community (or any other oncampus community for that matter). Student L, on the other hand, spent her first 3 years as a business major before changing to physics for years 4-7. It is notable that, while in business, Student L also picked up friends and study partners on a class-by-class basis. (This mirrors Student S's experience of an inconsistent peer group followed by attrition from her major.) Yet when Student L entered physics, she quickly integrated herself in the physics community. She spent a great deal of time in the Physics Learning Center, both studying and socializing with a consistent peer group on a daily basis for 3 years. She joined the campus Society of Physics Students (SPS) - whose membership significantly overlapped with PLC regulars - and eventually served in a leadership position there. She also taught as an LA for 6 semesters, engaging even more deeply with the physics department. Furthermore, she participated in several non-physics extracurricular groups on campus. In short, Student L became extremely involved with both the physics community and the social scene of the university at large. She explicitly attributed her success to the social support she received from her peers in SPS and the PLC, saying that they "helped me out so much" and "made it easier to deal with everything."

\section{B. Cross-case analysis}

Table I shows factors related to the students' engagement and persistence from two modules: Interactions with Physics Community and Environment/Barriers. In our analysis we look at how different factors affected the interviewed students' persistence and how it relates to our theoretical framework.

Commuting to school is traditionally considered to be a hindrance or pull factor [3]. Both students in our study were commuters. Even though Student L resided on campus for one year, we expect that commuting for most of their aca- 
demic careers would affect both students similarly. Moreover, even though Student S left the physics major, she remained at FIU in a different program. However, since $90 \%$ of FIU students commute, there is a culture of commuting that may lessen its effect. Thus, we cannot make definitive claims about whether commuting actually affected these students' persistence.

Usually, work responsibility is also considered a pull factor. However, participation in the LA program at FIU, which can be thought of as a special case of the work responsibility, has been found to actually have a positive effect on persistence $[12,13]$. Our study corroborates these previous results: working as an LA may help persistence by generating interactions with physics department personnel at multiple levels, including faculty members, graduate students, fellow undergraduate LA's, and undergraduate students attending courses. Student S, who ultimately left physics altogether, admitted that being a part of the LA program was the reason she actually re-considered the physics education program in the first place.

An emergent theme we identified is the importance of a consistent, long-term peer group. While Nora does mention peer group interactions, we found that both students ultimately left degree programs while interacting with peers on only a short-term basis. Student L attributes much of her success in physics to her long-term peer group, with whom she interacted both academically and socially. She even goes one step further and explicitly contrasts her inconsistent peer interactions in the business major with her daily interactions in physics. We believe that involvement in a consistent, longterm community is essential to fostering meaningful social integration that, ultimately, provides the support students need overcome their day-to-day struggles.

Related to the consistent peer group is the informal social support that a long-term community can offer. Encouragement from friends and a sense of shared struggle were vital to Student L's overcoming her doubts about continuing in physics. In contrast, Student S's lack of close friends at FIU denied her access to on-campus peer support that we speculate may have benefited her.

\section{CONCLUSIONS AND FUTURE WORK}

We found certain aspects of students' experiences to be particularly important for their persistence as physics majors. While Student S entered school with a pre-declared physics major and valued collaborative learning with classmates, working off-campus for 25 hours per week inhibited building a strong community of peers. The lack of consistent peer group may have further enabled attrition from the physics major in favor of a different degree program. We interpret this result to imply that, while Student $\mathrm{S}$ integrated well academically (at least on a per-class basis as indicated), she did not integrate socially with peers - she did not meaningfully immerse herself into the social fabric of the department or university. Student L, on the other hand, considered having a consistent peer group of support to be especially important for her persistence. Her peers helped her bridge the divide between academic and social interactions. Homework help, study partners, and "hang[ing] out" together were all important aspects of her social engagement.

We note that the factors presented in this paper are preliminary, based only on the pilot analysis of two students. We expect that these categories might expand, be resorted, or otherwise change upon inclusion of more interview data. Moreover, as mentioned earlier, we identified multiple factors organized into several modules that are beyond the scope of this paper. Future work, including analysis of additional interviews and cross-checking between factors and modules, may shed new light on the interpretation of our findings.

\section{ACKNOWLEDGMENTS}

The authors would like to thank Idaykis Rodriguez for insightful conversations early in the analysis process. This work was supported by NSF PHY 1344247.
[1] STEM Transformation Institute: Overview https://stem.fiu. edu/overview/ [Accessed Sep. 29, 2017]

[2] V. Tinto, Rev. Educ. Res. 45 (1975).

[3] A. Nora, in The Majority in the Minority: Expanding the representation of Latina/o faculty, administrators and students in higher education, edited by J. Castellanos \& L. Jones (Stylus Publishing, LLC, Sterling, VA, 2003).

[4] F. Marton, in Qualitative Research in Education: Focus and Methods, edited by R. R. Sherman \& R. B. Webb (The Falmer Press, Philadelphia, PA, 1988).

[5] V. Tinto, J. Coll. Stud. Ret. 8, 1 (2006).

[6] G. Crisp, A. Nora, and A. Taggart, Am. Educ. Res. J. 46 (2009).

[7] J. A. Fredricks, P.C. Blumenfeld, and A. H. Paris, Rev. Educ. Res. 74, 1 (2004).

[8] A. W. Astin, J. College Student Persistence 25 (1984).
[9] L. Wolf-Wendel, K. Ward, and J. Kinzie, J. Coll. Stud. Dev. 50, 4 (2009).

[10] First Generation Scholarship Program: Fast Facts. http:// firstgen.fiu.edu/index.php/about/ [Accessed May 9, 2017]

[11] US News: America's Best Colleges, http://colleges.usnews. rankingsandreviews.com/best-colleges/fiu-9635] [Accessed May 9, 2017]

[12] E. Brewe, L. H. Kramer, and G. E. O'Brien, AIP Conf. Proc. 1289, 85 (2010).

[13] R. M. Goertzen, E. Brewe, and L. Kramer, Int. J. Sci. Educ. 35, 2 (2013).

[14] E. Brewe, L. Kramer, and V. Sawtelle, Phys. Rev. ST Phys. Educ. Res. 8 (2012). 